\title{
Monitoring the Static Vehicle Load on a Heavy Goods Vehicle
}

\author{
Ivor Humphreys, Paul McDonald, Margaret O’Mahony and Dermot Geraghty \\ School of Engineering \\ Trinity College Dublin \\ Ireland \\ Email: tgerghty@tcd.ie
}

\begin{abstract}
Static vehicle load can be calculated accurately from measurements of axle strain. This paper describes, in outline form, a series of laboratory and field tests on an instrumented heavy goods vehicle. The vehicle was instrumented in the laboratory and an extensive series of calibration tests performed to establish the relationship between measurements of axle strain and vehicle load; this testing regime was also used to validate the instrumentation. A field trial was then conducted with the vehicle being returned to service and axle strain measurements being recorded continuously. Estimates of the static load were then validated against daily weigh-bridge measurements. The system has been in continuous service for several years now and has proven to be a robust and accurate method of calculating static vehicle load which can be easily installed on a vehicle at relatively low cost. When combined with a vehicle tracking unit real time load data can be made available to a fleet operator and on the spot load data displayed to the driver. Inadvertent overloading, with all its implications for safety and road damage, can thus be avoided.
\end{abstract}

\section{INTRODUCTION}

Overloading of HGVs, whether deliberate or inadvertent, is of major concern to those authorities charged with road maintenance since the road damage attributed to heavy goods vehicles is many orders of magnitude greater than that due to domestic automobiles. Tests have shown that increasing the load on a heavy goods vehicle (HGV) from 10 to 11 tonnes can increase the road damage by $46 \%$ [1]. Additionally there are serious safety implications with overloaded HGVs. Overloading impairs braking capability, increases the stresses on tyres causing them to wear more rapidly and increasing the danger of failure and may render the vehicle unstable particularly in cornering situations such as at roundabouts. In an enforcement campaign conducted in the UK from April 2002 to March 2003 57,000 HGVs were stopped and weighed. Of these 3,700 $(7.5 \%)$ were found to be overloaded and penalised. In the same period, 7006 light goods vehicles (LGVs) were weighed and $21 \%$ were found to be overloaded. Marchadour [2] indicates the scale of the problem in attempting enforcement of vehicle overloading regulations in France. In France in 2006 there were $450,000 \mathrm{HGVs}$ operating on its road network. Given the number of vehicles enforcement is a difficult task. The number of vehicles weighed per annum has declined four fold over the past 26 years while the number of vehicles in service has doubled. The maximum number of vehicles which can be weighed at present is less than 80,000 . HGVs were implicated in $8.9 \%$ of fatal accidents so there are serious safety concerns.

Hauliers and fleet operators are subject to random weight checks which generally involve diverting the vehicle to a weigh bridge so that the vehicle load can be measured or carrying out a roadside test using portable weigh pads. Breaches of the loading regulations can lead to fines and possibly the loss of a license. In many cases overloading may well be inadvertent as the driver may have no way of determining accurately if the vehicle is overloaded e.g. when removing debris from a construction site. In the United Kingdom the Vehicle and Operator Services Agency (VOSA) recommends that operators with fleets of HGVs install weighbridges. While some hauliers have their own weigh bridge installations and sophisticated instrumentation is available for on-vehicle load measurement it is expensive and difficult to justify for the majority of cases. Clearly, there is a need for a low cost vehicle mounted system which can provide an accurate measure of the vehicle load so that overloading is indicated clearly to the driver and the fleet operator. If combined with a vehicle tracking unit such a system provides the operator with a powerful tool not only for prevention of overloading but also for detection of fraud or potentially hazardous events such as load shifts in transit. It is not just simply the gross vehicle load that is of concern; there are also axle load limits.

In this paper we propose a system based on measurements of axle strain from which gross vehicle load and individual axle loads can be calculated. In the course of this work we modeled the strain distribution in the front and rear axles of a popular two axle $\mathrm{HGV}$ using finite element (FE) analysis under static and dynamic load conditions. The finite element models were used to identify high strain regions in the vehicle axles and to estimate its dynamic range. At this stage the FE model was only used as an indicator of likely regions where strain gauges could be installed. (Subsequently we tuned the FE model with experimental data from a laboratory test on the actual vehicle). In practice the actual strain levels were found to be quite low with a front axle strain-load sensitivity of approximately $11.5 \mu \epsilon / 100 \mathrm{~kg}$ and a rear axle strain-load sensitivity of approximately $3.5 \mu \epsilon / 100 \mathrm{~kg}$. We then designed and manufactured application specific instrumentation which could be easily and securely installed on the vehicle and which could be expected to withstand the rigours of a lengthy field 
trial. Weldable strain gauges were spot welded onto the front and rear axles at locations identified from a consideration of the FE data and other practical considerations and the custom instrumentation was then installed. We then conducted a static load calibration of the system in the laboratory which established the load-strain relationship and the repeatability of the strain measurements. From these measurements we were able to accurately calculate the static vehicle load regardless of the eccentricity of the load. Following the static calibration the vehicle was returned to service and six weeks later we repeated the static calibration. The results were found to be in close agreement.

The HGV was then returned to service with our instrumentation installed and continuous axle strain histories recorded on a flash memory key. In service this vehicle has its load weighed several times a day on a weigh bridge so the vehicle load is recorded. The operator provided us with this data which we were then able to correlate with the estimates of load made from the axle strain time histories. It proved very straightforward to identify periods in the strain time history when the vehicle was stationary and to estimate the load accurately. Our laboratory tests indicated that we should be able to calculate the load to within $1.5 \%$ of the true load; field tests were slightly less accurate at about $2.5 \%$. The remainder of this paper is organised as follows. In Section II we present a brief synopsys of related work. In Section III we present outline details of our instrumentation and describe our experimental procedure. In Section IV we present the results of our static calibration in the laboratory. The results from the field trial are presented in Section V. Finally, in Section VI we conclude with a discussion of the work and an outline of our plans to extend it.

\section{RELATED WORK}

Over the years various schemes for measuring the loads transmitted to the road surface by HGVs have been investigated and reported in the literature. These have been for research into road damage caused by HGVs or the effect on ride comfort of road surface roughness. In general, they have not been intended for use in determining the load carried by a vehicle at an arbitrary location on the road network i.e. vehicle based load measurement. In the main the systems reported are based on measurements of the deflection of tyres, the use of instrumented wheel hubs and strain gauging of axles. A review of the main methods employed prior to 1993 is presented in [1]. The number of vehicles instrumented for any particular study has been small and little attention has been paid to the problem of developing a low cost, robust, easily retrofitted system; this is the main thrust of our work. Apart from the methods described in [1] some researchers have proposed novel sensors for measurement of axle strain with this application in mind. A vehicle mounted load monitor was designed by Lacquet [4] using etched optical fibre sensors. The authors mounted the strain sensors on the front torsion bars and the rear axle of a Toyota Hi-Ace. They selected the positions of the sensors by trial and error. No attempt was made to model the axle using FE analysis or other method. The system proved accurate but needs more investigation to establish the long-term stability and durability of the sensor and in any event is not available. The application appears to have been chosen mainly to demonstrate the usefulness of the strain sensor primarily because of its resistance to electro-magnetic interference and its large gauge factor in comparison to conventional resistance strain gauges. Both of these are very important considerations as the small gauge factors of resistance strain gauges (2), combined with the very low strains developed in truck axles and the extremely noisy environment make accurate, high resolution measurements extremely difficult to achieve. An alternative system is presented in [3] based on measurement of wheel deflection, the premise being that the out-of-round shape of the wheel is almost linearly proportional to wheel load. Wheel deflection is measured using a capacitive displacement transducer. To determine the speed of rotation of the wheel, pins are placed at 45 degree intervals around the wheel rim. These pins are detected by the displacement transducer and appear as sharp spikes in the record of wheel displacement. The system is calibrated by unloading the wheel and rotating through 360 and recording the displacement transducer data to use as a reference. The wheel loading information can then be determined by subtracting the reference signal from a loading record either directly or using a method based on Fourier series extraction. The authors suggest that this method has possibilities as a cost effective research tool but faces some challenges before it could be commercialised.

Foster [5] showed both theoretically and experimentally that the static load on an axle could be accurately calculated from axle strain measurements. He demonstrated the principle in a laboratory test on the rear axle of a 1.5 tonne Bedford truck - a light goods vehicle. The strains measured in the axle were more than ten times the level we encountered in our experiments. The axle was mounted in a cradle, fitted with strain gauges mounted symmetrically about the axle vertical center line and loaded using a hydraulic cylinder. The applied load was measured using a calibrated load cell. In comparison our work was conducted on a fully instrumented vehicle and the strain gauge locations were determined by a finite element analysis of the vehicle axles rather than using the analytical approach used by Foster. Additionally, we conducted a lengthy field trial where vehicle load data was provided by a third party.

\section{Instrumentation AND EXPERIMENTAL PROCEDURE}

The test vehicle chosen was a MAN TGM 18.240, comprising a $\mathrm{C}$ class cab, standard frame and bed, HY-1350 hypoid rear axle and a VOK-08 non-drive front axle. This is a two axle truck with an 18 tonne capacity in service as a skip truck with a waste disposal company in Dublin. In normal service the entire truck and load is weighed on a weigh bridge at the operator's premises several times per day and the vehicle static load recorded. After installing and calibrating the instrumentation the vehicle was returned to normal service 
and the operator provided us with daily, data and time-stamped records of the weigh bridge data for the vehicle over a three month period. We were then able to correlate the weigh bridge data with our own date and time stamped axle strain histories and thus compare our estimates of load with the weigh bridge estimates. The viability of the system depends entirely on being able to accurately measure small changes in axle strain. Rear axle strain levels are particularly low of the order of $3.5 \mu \epsilon$ for a $100 \mathrm{~kg}$ change in load. Temperature performance now becomes a major factor with apparent strain due to temperature and bridge amplifier offset voltage drift with temperature becoming major contributors to the total error budget. Since robustness was also a key consideration we used weldable strain gauges rather than conventional foil gauges. These gauges are spot welded in position. Surface preparation is much simpler than with a conventional foil gauge and the installation time is much shorter. However, the cost of these gauges precluded the use of dummy gauges for temperature compensation. We opted to measure temperature at the gauge and correct numerically for apparent strain using calibration curves provided by the gauge manufacturer. Similarly we opted for the a quarter bridge configuration with a three wire connection to the gauge for lead wire resistance compensation. The gain stage is provided by an instrumentation amplifier with very high common mode rejection CMR (96 dB) and low input offset drift with temperature. The output offset is digitally programmable; this feature is used to map the bipolar bridge output of the bridge to the input range of the ADC. The instrumentation is mounted in an IP65 instrument case and mounted on the vehicle axle as close as is practicable to the strain gauges to limit gauge lead-wire lengths. One unit is required per axle. Both units record the axle strain data continuously and broadcast averaged strain data on an RS232 link. In laboratory bench tests and installed on a vehicle with the engine running the noise floor in the measurement system was measured at less than the equivalent of $1 \mu \epsilon$.

A series of static loading tests was carried out on the test vehicle. The truck axles were instrumented with weldable strain gauges, two gauges on the front axle and four on the rear axle. The installation locations for the gauges were identified using finite element analysis. The objectives of the tests were to establish the relationship between axle strain and axle load for static loading conditions, to determine the accuracy of the vehicle mounted instrumentation in measuring these strains, to establish the noise floor limit for the instrumentation and, most importantly, to establish the viability of the method as a vehicle mounted load measurement system. Tests were carried out with both uniformly distributed loads and offset or eccentric loads. Strain measurements were recorded while loading and unloading the vehicle. The test vehicle with incrementally loaded using calibrated 1-tonne weights in 1-tonne increments. Strain readings from the front and rear axles were recorded at each load increment. Simultaneously, the wheel loads were measured using four Hawkley ER540 portable weighing platforms. Each of these units has a $540 \mathrm{mmX} 390 \mathrm{~mm}$ aluminium plate onto which the wheel of the truck is positioned. An
RS232 output port transmits the data to a central control unit which displays the data for each wheel station and outputs the reading to a PC. Each of the units has a limit of $10,000 \mathrm{~kg}$ and has a traceable calibrated accuracy of $\pm 10 \mathrm{~kg}$.

\section{Static Calibration Results}

The system was calibrated using both uniformly distributed loads and offset loads. For the uniformly distributed loads the calibration weights were loaded onto the truck bed in 1 tonne increments and positioned about the longitudinal centerline of the truck in order to distribute the load between the near and off sides as evenly as possible. This provided near uniform load distribution across the bed of the truck with load sharing between the rear and front axles in the ratio 70:30 approximately. Weigh pad data were used to verify that the load distribution was uniform. Front and rear wheel loads, as indicated by the weigh pads, and near and off-side axle strain measurements were recorded for each load point. Fig. 1 and Fig. 2 show the results of calibration for uniformly distributed loading. Lines fitted to the data using linear least squares give $R^{2}$ values (coefficients of determination) of better than 0.99 for both the the front and rear axles. The standard error of the estimate of axle load from axle strain data was calculated at $\pm 1 \%$ for the rear axle and $\pm 1.5 \%$ for the rear axle. The aggregated values of front and rear axle strain are used to estimate gross vehicle load. The relationship between aggregated strain and gross vehicle load for uniformly distributed loading is shown in Fig. 3. The standard error of the estimate of gross vehicle load from total axle strain was calculated at $\pm 2 \%$. The front and rear axle strain sensitivities for uniformly distributed loads were $11.5 \mu \epsilon / 100 \mathrm{~kg}$ and $3.5 \mu \epsilon / 100 \mathrm{~kg}$ respectively.

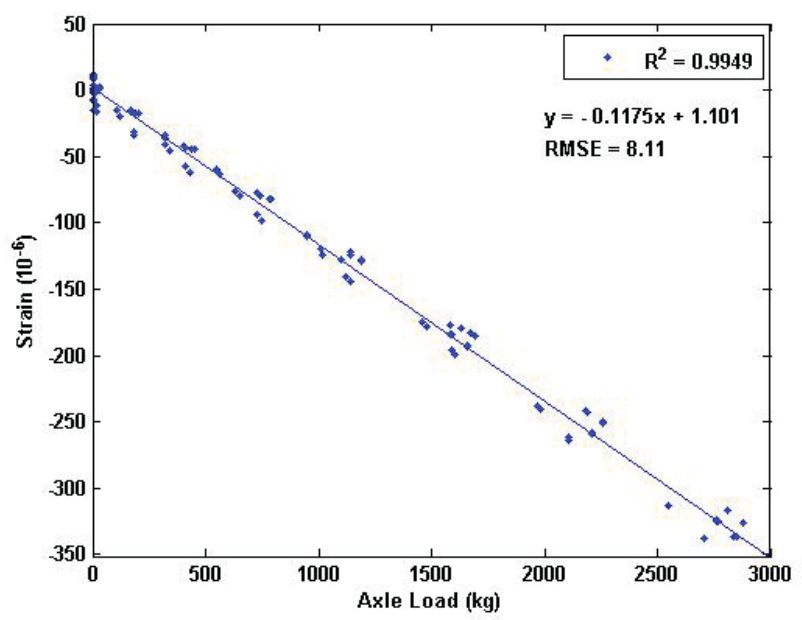

Fig. 1. Front axle aggregate strain versus axle load for uniformly distributed loading.

The calibration procedure was repeated for offset loads. The purpose of these tests was to establish that the total load on the vehicle could be established from axle strain measurements regardless of the eccentricity of the loading scheme.A series of eccentric loads $(0-4 t)$ were loaded on the truck bed, again 


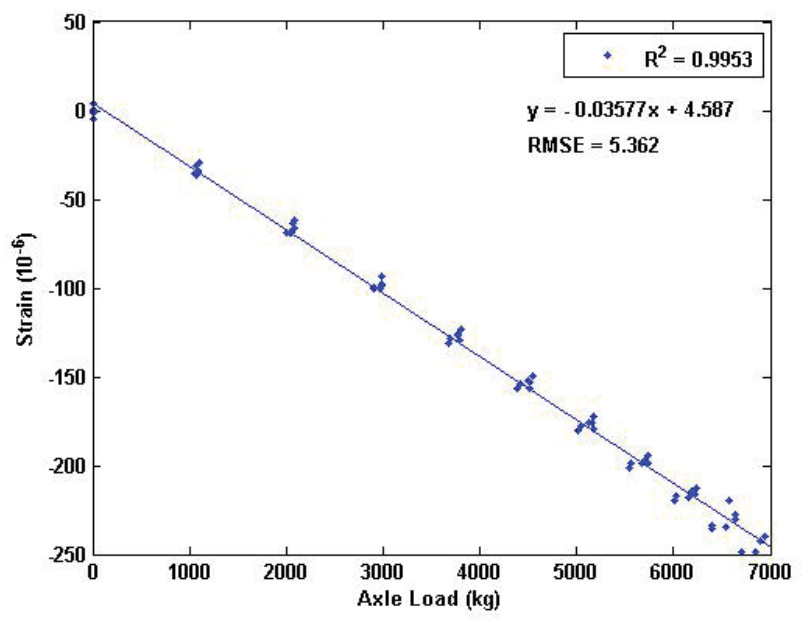

Fig. 2. Rear axle aggregate strain versus axle load for uniformly distributed loading

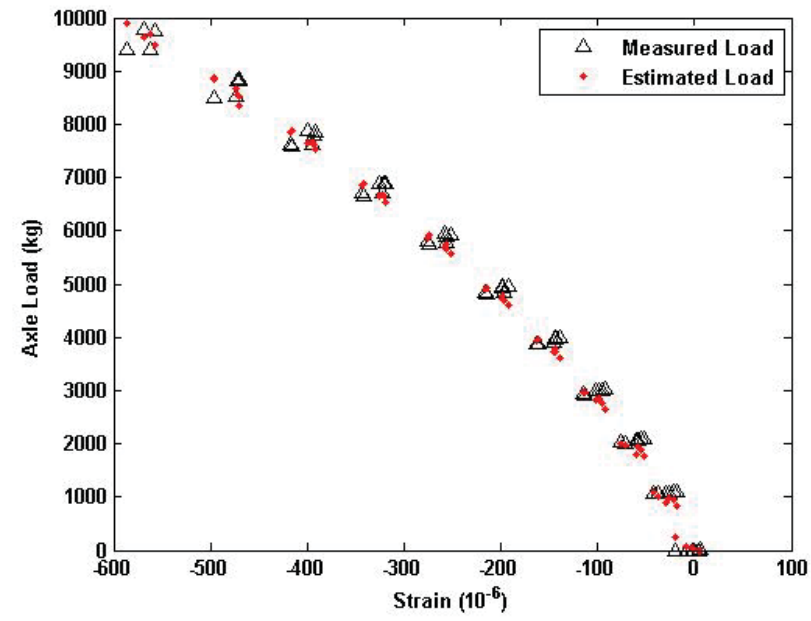

Fig. 3. Estimated gross vehicle load for uniformly distributed loading

in 1-t incrementt, offset to the near side of the vehicle. Again, strain readings were recorded at each load increment and decrement. The same process was repeated $(0-6 \mathrm{t})$ with the weights placed directly over the rear axle. The calibration data for the front and rear axles are shown in Fig. 4 and Fig. 5 respectively. Again, from the $R^{2}$ values the quality of the line fit is extremely good. The strain sensitivities for the offset loading case were almost identical to the uniformly distributed loading case. The estimated gross vehicle load is shown in Fig. 6.

\section{Field Trial Results}

The field trials have produced a wealth of data, a sample of which we provide here. Typical axle strain histories for both front and rear axles and presented in Fig.7 and Fig. 8 respectively. The 'quiet' periods correspond to times when the vehicle is actually on the weigh bridge and the operator is recording the load for billing purposes. This has been

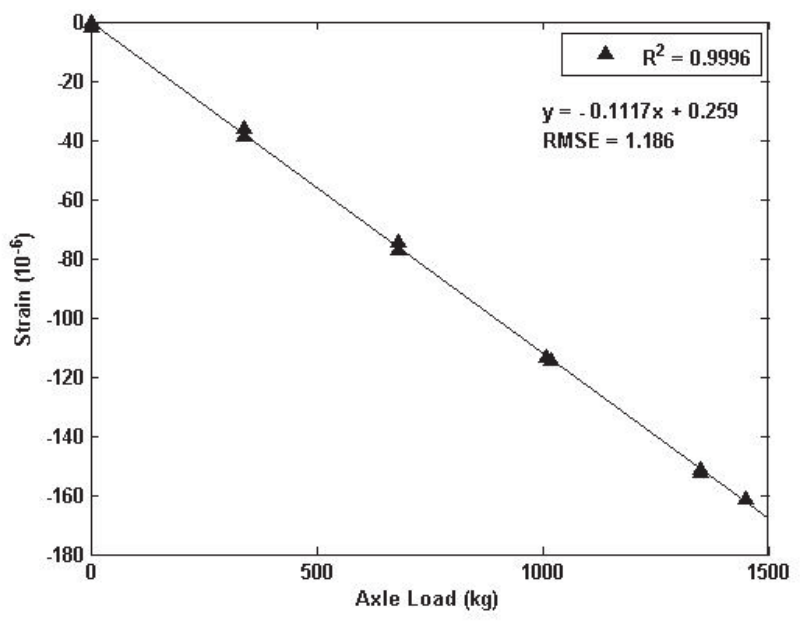

Fig. 4. Front axle aggregate strain versus axle load for offset loading.

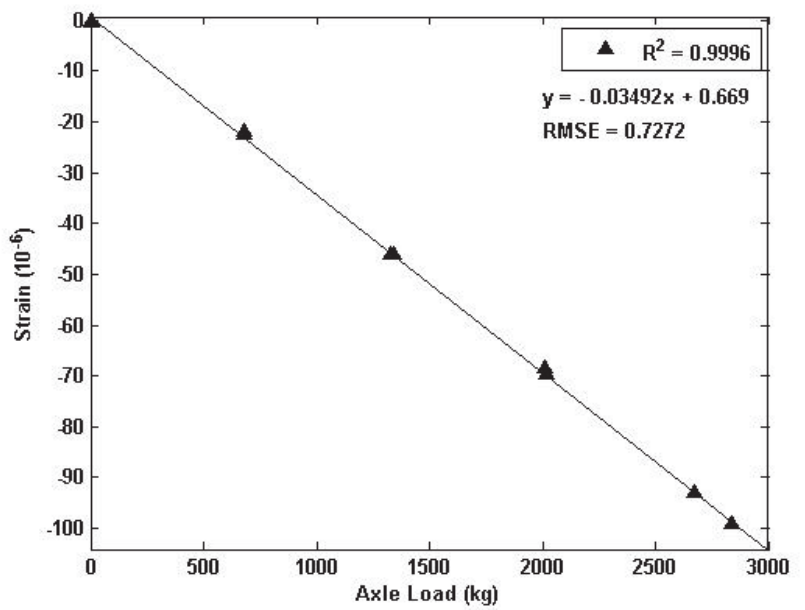

Fig. 5. Rear axle aggregate strain versus axle load for offset loading

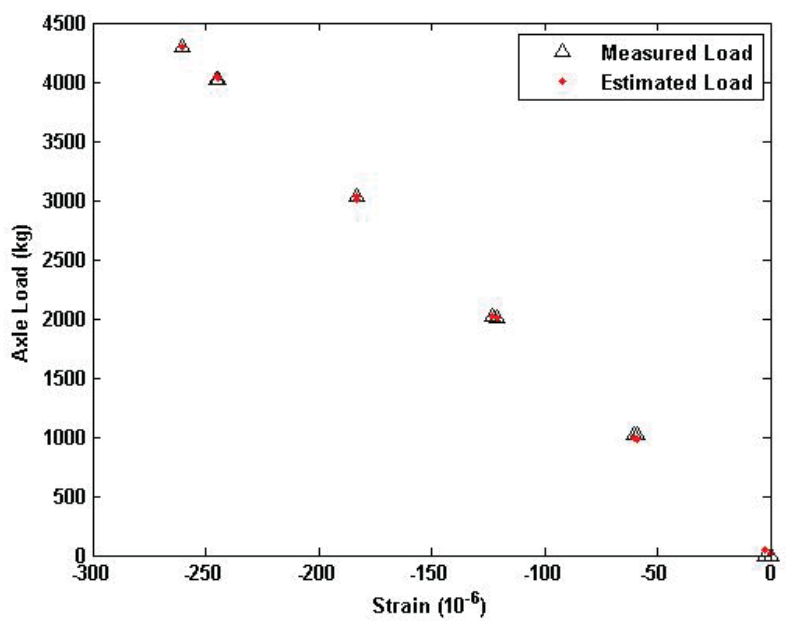

Fig. 6. Estimated gross vehicle load for offset loading 
confirmed using the operators GPS data and weighing records. Using the strain sensitivities calculated from the static calibration test it is possible to calculate the overall vehicle load and compare it with the weigh bridge data. The corresponding measurements are shown in Table X.

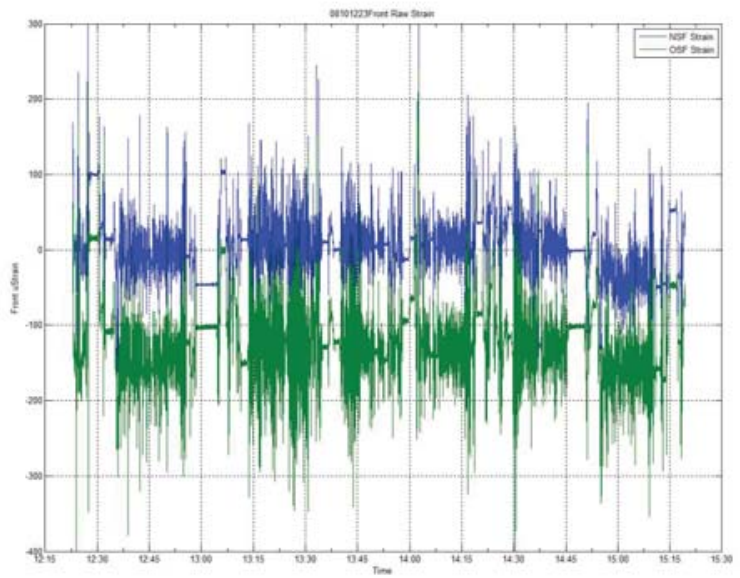

Fig. 7. Real Time Near Side and Off Side Front Axle Strain Data

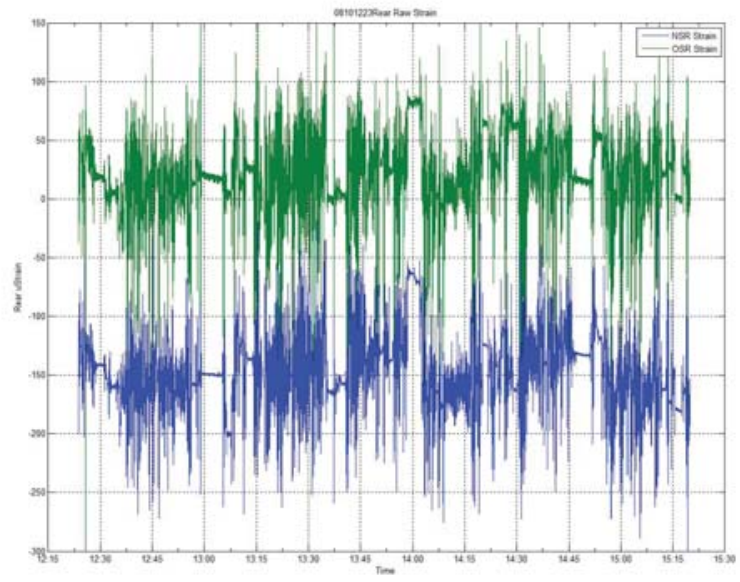

Fig. 8. Real Time Near Side and Off Side Rear Axle Strain Data

\section{CONCLUSIONS AND FURTHER WORK}

We have presented a method for calculating the static load on an HGV which is based on measurements of axle strain. We have designed and tested application specific instrumentation which has been proven capable of measuring very small axle strains. The instrumentation has been ruggedized and extensively tested in the laboratory. The system aas calibrated in the laboratory and then returned to service. The calibration was checked six weeks later by repeating the calibration procedure, following which a field trial was begun. The purpose of the field trial was to establish if the method could be used to measure reliably the static load on a vehicle under normal service conditions. The HGV we instrumented has been in continuous service with a waste disposal company and as part of its normal daily routine the static load on the vehicle is measured on a weigh bridge several times per day. The operator records the load data and the date and time of the measurement. Our on-board instrumentation stores date and time stamped axle strain histories and so we were able to compare the weigh bridge data with our estimates form these strain histories. We found the weigh bridge measurements and the calculated loads to be in close agreement. Note that load estimates are only calculated from the axle strain data when the vehicle is known or judged to be stationary i.e. during periods of relatively constant axle strain.

Axle strain histories were recorded continuously over a three month period and compared with the weigh bridge data. The instrumentation proved to be robust and reliable. We used weldable gauges in this application for ease of installation and for robustness. A drawback is that they are approximately ten times more expensive than conventional foil gauges. As a result there was an economic motivation to achieve accurate strain measurements with a minimum number of gauges two per axle. This proved feasible for the particular vehicle we selected for the field trial but heavier vehicles will likely have stiffer axles making the strain measurement problem more difficult. Future work will focus on extending the field trial to a wider variety of vehicles with different axle load capacities and configurations and on improvements to the instrumentation including an evaluation of possible alternatives to weldable gauges. The system has obvious benefits to operators who wish to avoid the safety hazards of overloading through self regulation when a weigh bridge is not readily available. The accuracy of the system has been measured at $2 \%$ of load. With a 10 tonne load this gives an uncertainty of $200 \mathrm{~kg}$. Where the objective is simply detection of an overload condition this error level is acceptable.

As a final note it is clear from the strain histories that it may be possible to estimate dynamic loads transmitted to the road surface from the axle strain measurements. As yet we have not fully evaluated this possibility but it is an ongoing area of investigation for us upon which we will report at a later stage.

\section{ACKNOWLEDGMENTS}

This work was supported by the Enterprise Ireland Innovation Partnership fund.

\section{REFERENCES}

[1] Cebon, D. Interaction Between Heavy Vehicles and Roads, SAE 930001, SP-951, 81p, 1993. Society of Automotive Engineers (LR Buckendale Lecture), ISBN 1-56091-336-3.

[2] Marchadour, Yves and Jacob, Bernard. Development and Implementation of a WIM Network for Implementation in France. In Proceedings of the International Conference on Heavy Vehicles,May 18-22, 2008. Paris, France

[3] Chang, W., Sverdlova, N., Sonmez, U. and Streit, D. (2000) Vehicle based weigh-in-motion system,Heavy Vehicle Systems, A Series of the international Journal of Vehicle Design, Vol. 7, No.s 2/3, pp. 205-218. 
[4] Lacquet, Beatrys M,. Swart, Pieter L and Kotze., Abraham P A low-cost on-board vehicle load monitor, Measurement Science and Technology, 7 (1996) 1761-1765.

[5] Foster, J.D.G., Measurement of Central or Offset Axle Load by AxleMounted Strain Gauges,Strain, (2003) 39, 21-26. 